(2) Open Access Full Text Article

ORIGINALRESEARCH

\title{
Provision of Palliative Care in Patients with COPD: A Survey Among Pulmonologists and General Practitioners
}

This article was published in the following Dove Press journal: International Journal of Chronic Obstructive Pulmonary Disease

Johanna MC Broese $\mathbb{D}^{1,2}$

Rianne MJJ van der Kleij (D)

Els ML Verschuur (D) ${ }^{2}$

Huib AM Kerstjens $\mathbb{D}^{3}$

Yvonne Engels $\mathbb{D}^{4}$

Niels H Chavannes (ID)

'Public Health \& Primary Care, Leiden University Medical Center, Leiden, the Netherlands; ${ }^{2}$ Lung Alliance Netherlands, Amersfoort, the Netherlands;

${ }^{3}$ Respiratory Medicine \& Tuberculosis, University of Groningen and University Medical Center Groningen, Groningen, the Netherlands; ${ }^{4}$ Anesthesiology, Pain \& Palliative Medicine, Radboud University Medical Center, Nijmegen, the Netherlands
Correspondence: Johanna MC Broese Public Health and Primary Care, Leiden University Medical Centre, Post Zone V0-P, Postbox 9600, Leiden, 2300 RC, the Netherlands

Email j.m.c.broese@lumc.nl
Introduction: Patients with advanced chronic obstructive pulmonary disease (COPD) experience significant symptom burden, leading to poor quality of life. Although guidelines recommend palliative care for these patients, this is not widely implemented and prevents them from receiving optimal care.

Objective: A national survey was performed to map the current content and organization of palliative care provision for patients with COPD by pulmonologists and general practitioners (GPs) in the Netherlands.

Methods: We developed a survey based on previous studies, guidelines and expert opinion. Dutch pulmonologists and GPs were invited to complete the survey between April and August 2019.

Results: 130 pulmonologists (15.3\%; covering $76 \%$ of pulmonology departments) and 305 GPs $(28.6 \%)$ responded. Median numbers of patients with COPD in the palliative phase treated were respectively 20 and 1.5 per year. $43 \%$ of pulmonologists and $9 \%$ of GPs reported some formalized agreements regarding palliative care provision. Physicians most often determined the start of palliative care based on clinical expertise or the Surprise Question. 31\% of pulmonologists stated that they often or always referred palliative patients with COPD to a specialist palliative care team; a quarter rarely referred. $79 \%$ of the respondents mentioned to often or always administer opioids to treat dyspnea. The topics least discussed were noninvasive ventilation and the patient's spiritual needs. The most critical barrier to starting a palliative care discussion was difficulty in predicting the disease course.

Conclusion: Although pulmonologists and GPs indicated to regularly address palliative care aspects, palliative care for patients with COPD remains unstructured and little formalized. However, our data revealed a high willingness to improve this care. Clear guidance and standardization of practice are needed to help providers decide when and how to initiate discussions, when to involve specialist palliative care and how to optimize information exchange between care settings.

Keywords: COPD, palliative care, advance care planning, surprise question, organization

\section{Introduction}

Chronic obstructive pulmonary disease (COPD) is the third leading cause of death worldwide. ${ }^{1}$ In advanced stages, COPD is associated with a significant symptom burden and poor quality of life. ${ }^{2}$ However, palliative care is often not provided to patients with COPD; discussions on palliative and end-of-life care rarely occur, ${ }^{3}$ and access to specialist palliative care is limited and late. ${ }^{4}$ This lack of palliative care is 
often attributed to the unpredictable disease course of COPD, making it hard to determine when to start palliative care. ${ }^{5}$

Consequently, physicians and policymakers increasingly acknowledge the need for better and timely palliative care provision for patients with COPD. ${ }^{6-8}$ In the Netherlands, various efforts have been deployed to improve quality and timing of palliative care for patients with life-limiting diseases, including COPD. A national multidisciplinary guideline on this topic was published in 2011. ${ }^{9}$ Furthermore, the Quality Framework Palliative Care was published in 2017, to incentivize implementation of palliative care in the Netherlands. ${ }^{10}$ The framework describes the essential elements needed to deliver high-quality palliative care. In this framework, no distinction is made between oncologic and non-oncologic diseases, following the WHO definition of palliative care. ${ }^{11}$

Previous studies have highlighted that the provision of palliative care to patients with COPD is unstructured and often limited to terminal care only. ${ }^{3,12}$ However, no study has yet examined the formalization and implementation of palliative care for patients with COPD in the Netherlands. Therefore, we developed a national survey to explore the current content and organization of palliative care for patients with COPD in primary and secondary care in the Netherlands.

\section{Materials and Methods \\ Design}

A national survey study was performed among pulmonologists and general practitioners (GPs) in the Netherlands, to examine both the primary and secondary palliative care provision to patients with COPD.

A

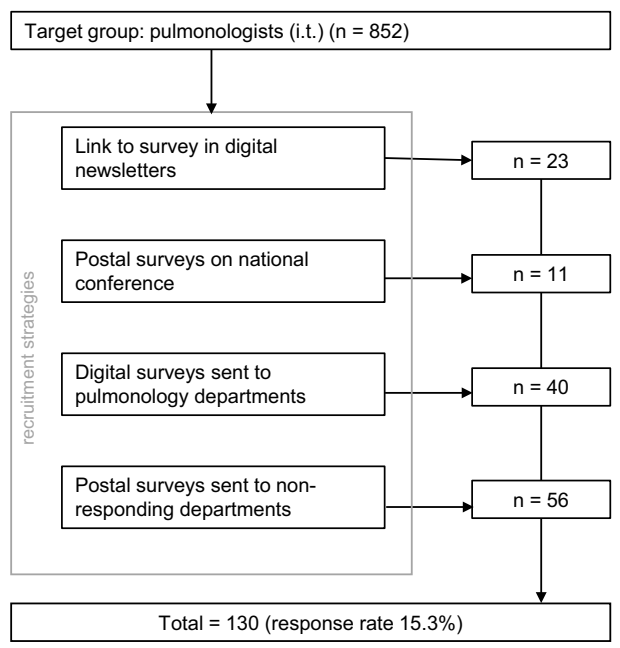

\section{B}

\section{Procedure}

Pulmonologists ( $\mathrm{n}=668)$ and pulmonologists in training (i.t.) $(n=184)$ registered by the Netherlands Association of Physicians for Lung Diseases and Tuberculosis (NVALT) were recruited via various and subsequent strategies between April and August 2019, to maximize participation (see Figure 1). Both a digital link to the online survey and a paper version was used. A link to the survey was disseminated via two subsequent digital newsletters of the NVALT, a digital newsletter of the Lung Alliance Netherlands (LAN) and via e-mails to each pulmonology department $(n=80)$ in the Netherlands. Also, pulmonologists visiting the annual Dutch pulmonologist conference were asked to complete a paper version of the survey. Subsequently, paper versions of the survey were sent to pulmonologists of departments of which no pulmonologist had responded yet.

GPs were recruited in two ways. First, postal surveys were sent to a random sample of 900 general practices in the Netherlands, obtained via the Netherlands Institute for Health Services Research (NIVEL). A reminder was sent to non-responding general practices after five weeks. Second, a digital link to the online survey was sent to 165 GPs via two GP expert advice networks on COPD/ asthma care (CAHAG) and palliative care (PalHag).

All responses received before August 21, 2019 were included in the analysis. The online data management system Castor EDC was used for data collection. Consent to participate was implied by responding to the survey. This study was approved by the Medical Ethics Committee of the Radboud University Medical Center (number 2019-5021).

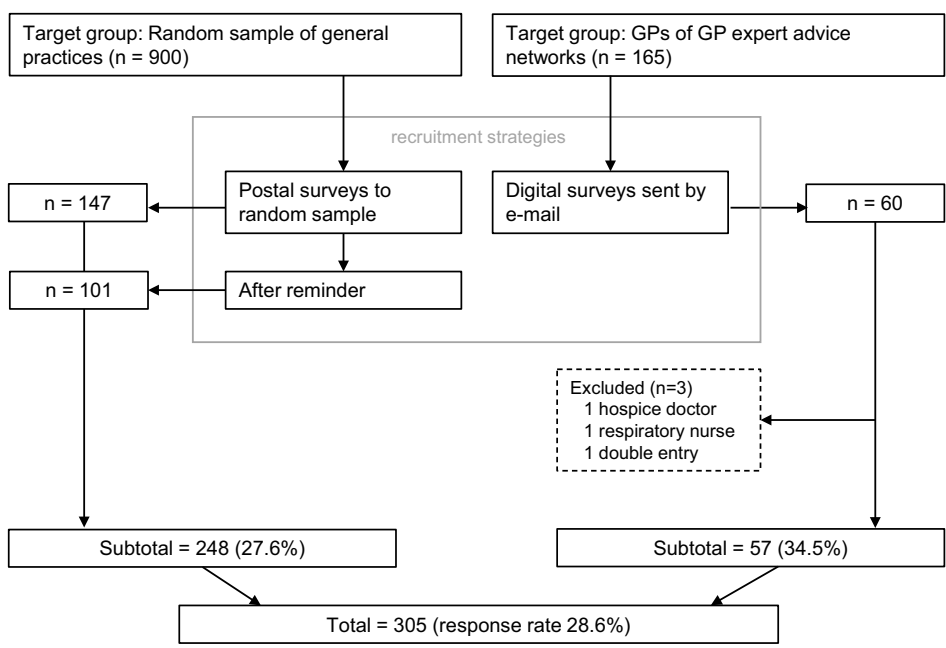

Figure I Flowchart of recruitment strategies and numbers of responding pulmonologists $(\mathbf{A})$ and general practitioners (B). Abbreviations: GP, general practitioner; i.t., in training. 


\section{Survey}

The survey was developed based on previous studies ${ }^{7,13,14}$ and national guidelines. ${ }^{9,10}$ In several cycles, we presented drafts of the survey to care providers and experts for adaptations and improvements. The survey started with an introductory text explaining the definition of palliative care and the palliative phase in COPD according to the national guideline ${ }^{9}$ (Supplementary Table S1). Then, questions were presented regarding the presence and plans for future development of any formalized agreements (i.e. a protocol or specific agreements) in their department or practice, and methods used to identify the palliative phase in patients with COPD. This was followed by a question on the level of experience with palliative care provision in COPD and a question on the estimated number of palliative patients with COPD the participant treated on average per year. Participants who had treated at least one such patient in the last year, were further inquired on the frequency of palliative care aspects provided and topics discussed in the previous year, and collaboration with and referral to other care providers. Answer options ranged from never to always on a 5-point Likert scale. Also, satisfaction with collaboration between pulmonologists and GPs was inquired. The next question of the survey was presented to all respondents and related to perceived barriers towards palliative care discussions; multiple answer entries were allowed on fourteen statements. Lastly, the following characteristics were questioned: age, gender, position, workplace, work experience and education.

\section{Statistical analysis}

All data were analyzed using descriptive statistics. For each item, all available data were used. Therefore, the total number of respondents varied per item. Noncontinuous variables were reported as frequencies. Answers in free text boxes were inductively coded and categorized using Atlas.ti. Differences between 1) pulmonologists and GPs and 2) physicians with and without any form of palliative care training were tested using the Mann-Whitney $U$-test for categorical variables (using 5 answer categories) and the Chi-square test for dichotomous variables. Differences were considered significant if $p<0.05$. Statistical analyses were performed with IBM SPSS Statistics, version 25.

\section{Results}

\section{Study participants}

In total, 130 pulmonologists (response rate of $15.3 \%$ ), including four pulmonologists in training, and 305 GPs (response rate of $28.6 \%$ ) responded to the survey between April and August 2019 (Figure 1). The responding pulmonologists were employed in 61 of 80 pulmonology departments $(76 \%)$ in the Netherlands. The median number of COPD patients treated in the palliative phase was on average 20 per year for pulmonologists and 1.5 per year for GPs (Table 1). In the last year, three pulmonologists and sixty-five GPs had not treated any palliative patient with COPD. Non-response per item was on average $12.9 \%$ in returned surveys of pulmonologists and $6.0 \%$ in those of GPs (Supplementary Table S1).

Table I Characteristics of participating pulmonologists and general practitioners

\begin{tabular}{|c|c|c|}
\hline & Pulmonologists $(n=130) n(\%)$ & General Practitioners $(n=305)$ n (\%) \\
\hline Age (years), mean (SD) & $46.0(8.9)$ & $49.4(9.6)$ \\
\hline Gender, male & $67(59.3)$ & $124(43.5)$ \\
\hline Work experience (years), mean (SD) & $13.0(8.8)$ & $17.8(9.7)$ \\
\hline \multicolumn{3}{|l|}{ Workplace } \\
\hline General hospital & $54(47.8)$ & N/A \\
\hline Top clinical hospital & $48(42.5)$ & \\
\hline University hospital & $8(7.1)$ & \\
\hline Other & $3(2.7)$ & \\
\hline Experience with PC provision in COPD* & $97(78.0)$ & $105(34.8)$ \\
\hline Median number of palliative patients with COPD treated yearly & 20 (range 0-1020) & 1.5 (range 0-30) \\
\hline Treated $\geq$ I palliative patient with COPD last year & $127(97.7)$ & $240(78.7)$ \\
\hline \multicolumn{3}{|l|}{ Education } \\
\hline Palliative care training, any & $36(35.7)$ & $93(34.6)$ \\
\hline Specialized training in palliative care & $2(1.7)$ & $22(7.4)$ \\
\hline Specialized training in asthma/COPD & $\mathrm{N} / \mathrm{A}$ & $25(8.9)$ \\
\hline
\end{tabular}

Note: *Respondents with answers "a reasonable amount" and "a lot". 


\section{Structure of palliative care}

Fifty-six pulmonologists (45.9\%) reported that there were no formalized agreements on the palliative care provision to patients with COPD at their department and thirteen (10.7\%) indicated did not know. Fifty-two pulmonologists (43.4\%) reported that there were formalized agreements; most detailed the possibility to involve a specialist palliative care team $(\mathrm{n}=22 ; 18.0 \%)$. Sixteen pulmonologists $(13.2 \%)$ indicated a hospital-specific care pathway was present, five of these were palliative sedation protocols or dying care pathways. Other agreements covered advance care planning discussions $(\mathrm{n}=9 ; 7.4 \%)$ and agreements on e.g. scoring symptoms or starting morphine $(6 ; 5.0 \%)$. Half of the pulmonologists $(\mathrm{n}=60 ; 50.4 \%)$ indicated that their department had plans to establish formalized agreements in the future; fourteen $(11.9 \%)$ referred to the development of a new protocol and eight (6.8\%) to the adaptation of an existing protocol or care pathway.

The majority of GPs $(n=269 ; 89.4 \%)$ reported that there were no formalized agreements. Twenty-six GPs $(8.6 \%)$ reported that there were; most of them covered patient support by the GP, a practice nurse or palliative care nurse $(\mathrm{n}=11 ; 3.7 \%)$, and participation in a PaTz (palliative homecare) group $(\mathrm{n}=8 ; 2.7 \%)$. No protocols were reported. Thirty-five GPs (11.7\%) indicated to have plans to formalize palliative care in COPD in the future, of which eight indicated they planned to establish a protocol.

\section{Identification of the palliative phase in patients with COPD}

As reported by our respondents, the palliative phase in patients with COPD was most often determined based on clinical expertise or by using the Surprise Question (SQ) (Figure 2). The SQ reads "Would I be surprised if this patient were to die in the next twelve months?" More pulmonologists indicated to use the SQ than GPs $(76.9 \%$ vs. $56.7 \%, p<0.001)$. Many GPs also determined the palliative phase based on information transfer or transfer of care from the pulmonologist to the GP. Thirteen participants $(3.1 \%)$ indicated not to discern a palliative phase.

\section{Symptom management}

Most respondents $(\mathrm{n}=283 ; 79.3 \%)$ reported to often or always administer opioids to treat dyspnea (Table 2). No significant differences in frequency were observed between pulmonologists and GPs nor between those with and without palliative care training. GPs prescribed pharmacological treatment for anxiety and depression more often than pulmonologists $(p=0.046)$. Physicians with palliative care training more frequently provided non-

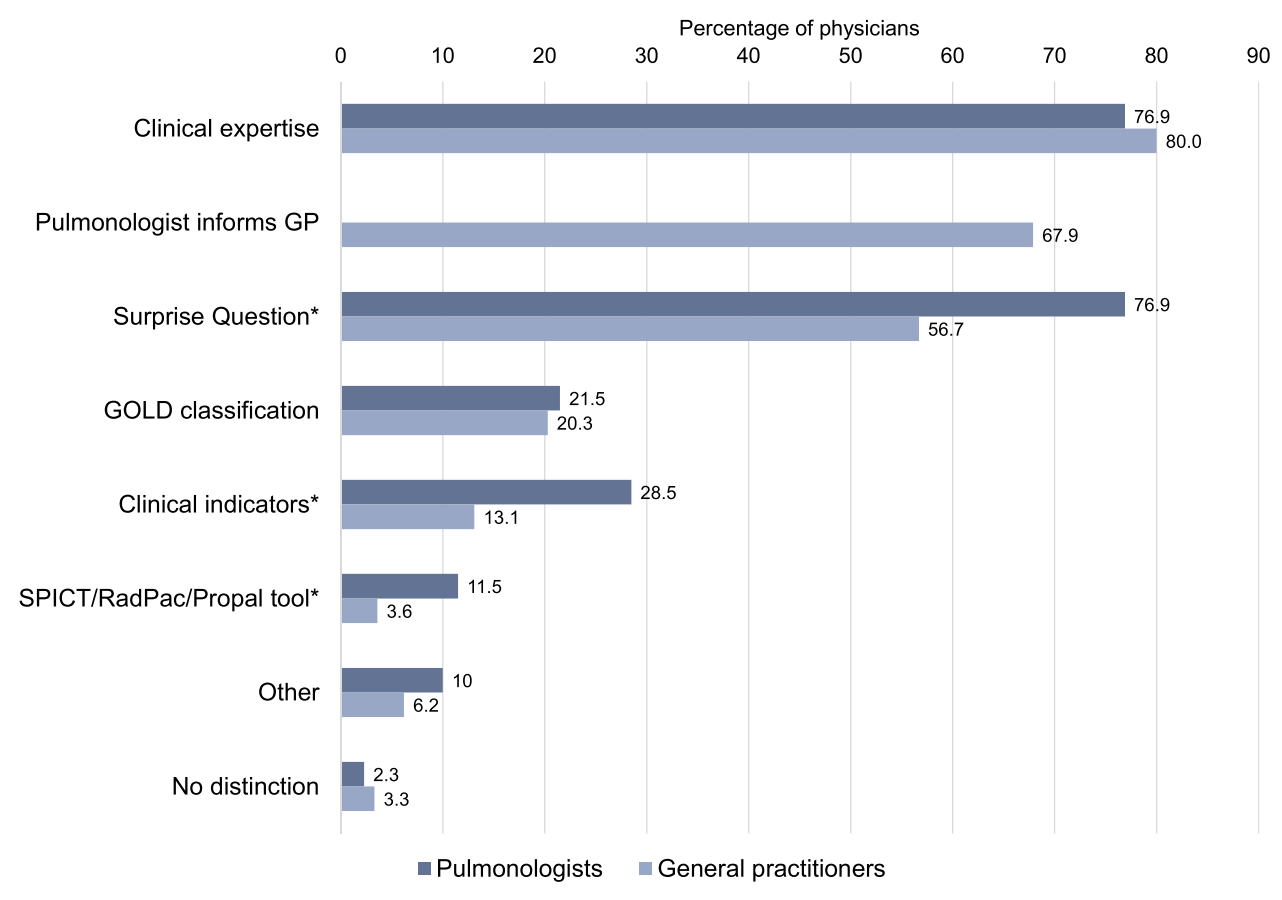

Figure 2 Methods used by pulmonologists and general practitioners to identify the palliative phase in patients with COPD. *Significant difference $(p<0.05$ using Chi-square test). 
Table 2 Symptom management in patients with COPD in the palliative phase that respondents had treated in the previous year, and comparison of pulmonologists and general practitioners

\begin{tabular}{|c|c|c|c|c|c|}
\hline & & All respondents & Pulmonologists & GPs & $P$-value ${ }^{a}$ \\
\hline \multirow[t]{5}{*}{ Dyspnea using opioids } & Never & $6(1.7)$ & $0(0.0)$ & $6(2.5)$ & 0.077 \\
\hline & Rarely & $8(2.2)$ & $4(3.3)$ & $4(1.7)$ & \\
\hline & Sometimes & $60(16.8)$ & $18(15.0)$ & $42(17.7)$ & \\
\hline & Often & $202(56.6)$ & $84(70.0)$ & II 8 (49.8) & \\
\hline & Always & $81(22.7)$ & 14 ( 11.7$)$ & $67(28.3)$ & \\
\hline \multirow[t]{5}{*}{ Dyspnea using non-pharmacological treatment } & Never & $12(3.5)$ & I (0.9) & II (4.8) & 0.142 \\
\hline & Rarely & $35(10.1)$ & $9(7.8)$ & $26(11.4)$ & \\
\hline & Sometimes & $112(32.5)$ & $37(31.9)$ & $75(32.8)$ & \\
\hline & Often & $149(43.2)$ & $58(50.0)$ & $91(39.7)$ & \\
\hline & Always & $37(10.7)$ & II (9.5) & $26(11.4)$ & \\
\hline \multirow[t]{5}{*}{ Pain using opioids } & Never & $24(6.8)$ & $6(5.1)$ & $18(7.7)$ & 0.625 \\
\hline & Rarely & $84(23.9)$ & $26(22.2)$ & $58(24.8)$ & \\
\hline & Sometimes & $146(4 \mid .6)$ & $54(46.2)$ & $92(39.3)$ & \\
\hline & Often & $82(23.4)$ & $26(22.2)$ & $56(23.9)$ & \\
\hline & Always & $15(4.3)$ & $5(4.3)$ & $10(4.3)$ & \\
\hline \multirow[t]{5}{*}{ Anxiety/depression using pharmacological treatment } & Never & $23(6.6)$ & $9(7.8)$ & $14(6.0)$ & 0.046 \\
\hline & Rarely & $50(14.4)$ & $22(19.1)$ & $28(12.1)$ & \\
\hline & Sometimes & I55 (44.7) & $51(44.3)$ & $104(44.8)$ & \\
\hline & Often & III (32.0) & $31(27.0)$ & $80(34.5)$ & \\
\hline & Always & $8(2.3)$ & $2(1.7)$ & $6(2.6)$ & \\
\hline \multirow[t]{5}{*}{ Anxiety/depression using non-pharmacological treatment } & Never & $20(5.9)$ & $5(4.4)$ & $15(6.6)$ & 0.665 \\
\hline & Rarely & $63(18.5)$ & 25 (2I.9) & 38 (16.7) & \\
\hline & Sometimes & 149 (43.7) & $48(42.1)$ & IOI (44.5) & \\
\hline & Often & $96(28.2)$ & $34(29.8)$ & $62(27.3)$ & \\
\hline & Always & $13(3.8)$ & $2(1.8)$ & II (4.8) & \\
\hline
\end{tabular}

Notes: Data are expressed as absolute values and percentages. ${ }^{a}$-values based on Mann-Whitney U-test.

pharmacological treatment for anxiety and depression ( $p=0.030$ for pulmonologists and $p=0.011$ for GPs) than those without (Supplementary Table S2).

\section{Doctor-patient-family communication}

Almost all respondents indicated to often or always discuss palliative treatment options for dyspnea $(n=307$; 92.1\%) (Table 3). Approximately a third of respondents rarely or never discussed non-invasive ventilation $(\mathrm{n}=112$; $33.4 \%$ ) or spiritual needs ( $\mathrm{n}=92 ; 27.8 \%)$. GPs discussed seven topics more frequently than pulmonologists. Pulmonologists discussed non-invasive ventilation more often than GPs. Pulmonologists with palliative care training discussed six topics more often than pulmonologists without training (Supplementary Table S3): fear of choking $(p=0.015)$, fear of death/dying $(p=0.025)$, preferred place of death $(p=0.005)$, spiritual needs $(p=0.007)$, caregiver burden $(p=0.003)$ and goals of care $(p=0.020)$. Within GPs, no differences were found between those with and without palliative care training.

Most GPs ( $\mathrm{n}=200 ; 91.7 \%)$ mentioned that, in the past year, they had often or always discussed treatment preferences at home; a minority $(\mathrm{n}=46 ; 21.6 \%)$ discussed them at the practice. More than half $(\mathrm{n}=116 ; 53.5 \%)$ often or always discussed these preferences shortly after a hospitalization for an acute exacerbation. Of the pulmonologists, $41.3 \%(n=43)$ stated to have often or always discussed preferences when patients visited the emergency department, 66.3\% $(\mathrm{n}=69)$ during hospitalization and $52.4 \%(\mathrm{n}=54)$ at the outpatient clinic. More GPs $(\mathrm{n}=105$; $48.4 \%)$ than pulmonologists $(\mathrm{n}=23 ; 21.9 \%)$ mentioned that they often or always planned an appointment specifically for these discussions; $24.4 \%$ of the GPs $(n=53)$ and $48.6 \%$ of pulmonologists $(n=51)$ never or rarely did so. The 
Table 3 Frequency of topics discussed by respondents in the previous year, and comparison of pulmonologists and general practitioners

\begin{tabular}{|c|c|c|c|c|c|}
\hline & & All respondents & Pulmonologists & GPs & $P$ value $^{a}$ \\
\hline \multirow[t]{5}{*}{ Disease course and incurability } & Never & $2(0.6)$ & $0(0.0)$ & $2(0.9)$ & 0.014 \\
\hline & Rarely & $4(1.2)$ & $2(1.8)$ & $2(0.9)$ & \\
\hline & Sometimes & $39(11.4)$ & $13(11.4)$ & $26(11.4)$ & \\
\hline & Often & $162(47.4)$ & $67(58.8)$ & $95(4 I .7)$ & \\
\hline & Always & $135(39.5)$ & $32(28.1)$ & $103(45.2)$ & \\
\hline \multirow[t]{5}{*}{ Life expectancy } & Never & $9(2.7)$ & $0(0.0)$ & $9(4.0)$ & 0.050 \\
\hline & Rarely & $30(8.9)$ & $14(12.5)$ & $16(7.1)$ & \\
\hline & Sometimes & $98(29.0)$ & $41(36.6)$ & $57(25.2)$ & \\
\hline & Often & $133(39.3)$ & $39(34.8)$ & $94(4 \mid .6)$ & \\
\hline & Always & $68(20.1)$ & $18(16.1)$ & $50(22.1)$ & \\
\hline \multirow[t]{5}{*}{ Fear of choking } & Never & $\mathrm{I}(0.3)$ & $0(0.0)$ & $\mathrm{I}(0.4)$ & 0.065 \\
\hline & Rarely & $10(3.0)$ & $3(2.7)$ & $7(3.1)$ & \\
\hline & Sometimes & $53(15.7)$ & $18(16.1)$ & $35(15.5)$ & \\
\hline & Often & $169(50.0)$ & $67(59.8)$ & $102(45.1)$ & \\
\hline & Always & $105(31.1)$ & $24(2 I .4)$ & $81(35.8)$ & \\
\hline \multirow[t]{5}{*}{ Fear of death/dying } & Never & $3(0.9)$ & $\mathrm{I}(0.9)$ & $2(0.9)$ & $<0.001$ \\
\hline & Rarely & $13(3.9)$ & $7(6.3)$ & $6(2.7)$ & \\
\hline & Sometimes & $64(19.3)$ & $37(33.0)$ & $27(12.3)$ & \\
\hline & Often & $149(44.9)$ & $46(4 I . I)$ & $103(46.8)$ & \\
\hline & Always & $103(31.0)$ & $21(18.8)$ & $82(37.3)$ & \\
\hline \multirow[t]{5}{*}{ Advantages and disadvantages of life sustaining treatments } & Never & $0(0.0)$ & $0(0.0)$ & $0(0.0)$ & 0.456 \\
\hline & Rarely & $7(2.1)$ & I (0.9) & $9(8.0)$ & \\
\hline & Sometimes & $30(9.0)$ & $6(2.7)$ & $21(9.5)$ & \\
\hline & Often & $149(44.7)$ & $58(5 \mid .8)$ & $91(4 I .2)$ & \\
\hline & Always & $147(44.1)$ & $44(39.3)$ & $103(46.6)$ & \\
\hline \multirow[t]{5}{*}{ Advantages and disadvantages of non-invasive ventilation } & Never & $56(16.7)$ & $0(0.0)$ & $56(25.2)$ & $<0.001$ \\
\hline & Rarely & $56(16.7)$ & $5(4.4)$ & $51(23.0)$ & \\
\hline & Sometimes & $88(26.3)$ & $28(24.8)$ & $60(27.0)$ & \\
\hline & Often & $93(27.8)$ & $60(53.1)$ & $33(14.9)$ & \\
\hline & Always & $42(12.5)$ & $20(17.7)$ & $22(9.9)$ & \\
\hline \multirow[t]{5}{*}{ Desirability of hospitalization for acute exacerbation } & Never & $4(1.2)$ & I (0.9) & $3(1.3)$ & $<0.001$ \\
\hline & Rarely & $14(4.2)$ & II (9.8) & $3(1.3)$ & \\
\hline & Sometimes & $64(19.0)$ & $39(34.8)$ & $25(11.2)$ & \\
\hline & Often & $158(47.0)$ & $52(46.4)$ & $106(47.3)$ & \\
\hline & Always & $96(28.6)$ & $9(8.0)$ & $87(38.8)$ & \\
\hline \multirow[t]{5}{*}{ Palliative treatment options for dyspnea (e.g. morphine) } & Never & $0(0.0)$ & $0(0)$ & $0(0)$ & $<0.001$ \\
\hline & Rarely & $\mathrm{I}(0.3)$ & $0(0)$ & I $(0.4)$ & \\
\hline & Sometimes & $25(7.5)$ & $10(9.1)$ & $15(6.7)$ & \\
\hline & Often & $161(48.3)$ & $73(66.4)$ & $88(39.5)$ & \\
\hline & Always & $146(43.8)$ & $27(24.5)$ & $119(53.4)$ & \\
\hline \multirow[t]{5}{*}{ Preferred place of death } & Never & $6(1.8)$ & $5(4.6)$ & I (0.4) & $<0.001$ \\
\hline & Rarely & $28(8.4)$ & $21(19.3)$ & $7(3.1)$ & \\
\hline & Sometimes & $61(18.3)$ & $36(33.0)$ & $25(11.2)$ & \\
\hline & Often & II4 (34.2) & $34(3 \mid .2)$ & $80(35.7)$ & \\
\hline & Always & $124(37.2)$ & $13(11.9)$ & III (49.6) & \\
\hline
\end{tabular}

(Continued) 
Table 3 (Continued).

\begin{tabular}{|l|c|c|c|c|c|}
\hline & & All respondents & Pulmonologists & GPs & P value \\
\hline Spiritual and existential needs & Never & $25(7.6)$ & $15(13.8)$ & $10(4.5)$ & $<0.001$ \\
& Rarely & $67(20.2)$ & $40(36.7)$ & $27(12.2)$ \\
& Sometimes & $119(36.0)$ & $36(33.0)$ & $83(37.4)$ \\
& Often & $85(25.7)$ & $14(12.8)$ & $71(32.0)$ \\
& Always & $35(10.6)$ & $4(3.7)$ & $31(14.0)$ \\
\hline Caregiver burden & Never & $3(0.9)$ & $1(0.9)$ & $1(0.9)$ & $<0.001$ \\
& Rarely & $25(7.6)$ & $20(18.5)$ & $5(2.2)$ \\
& Sometimes & $97(29.3)$ & $51(47.2)$ & $46(20.6)$ \\
& Often & $147(44.4)$ & $32(29.6)$ & $115(51.6)$ \\
& Always & $59(17.8)$ & $4(3.7)$ & $55(24.7)$ \\
\hline Goals of care & Never & $21(6.4)$ & $1(0.9)$ & $20(9.0)$ & 0.702 \\
& Rarely & $35(10.7)$ & $12(11.2)$ & $23(10.4)$ \\
& Sometimes & $131(39.9)$ & $49(45.8)$ & $82(37.1)$ \\
& Often & $102(31.1)$ & $35(32.7)$ & $67(30.3)$ \\
& Always & $39(11.9)$ & $10(9.3)$ & $29(13.1)$ \\
\hline
\end{tabular}

Notes: Data are expressed as absolute values and percentages. ${ }^{a}$-values based on Mann-Whitney $U$-test.

majority of pulmonologists $(\mathrm{n}=92 ; 86.8 \%)$ and GPs $(\mathrm{n}=159 ; 72.3 \%)$ reported that they never or rarely discussed preferences with a family member without the patient being present. Bereavement support to relatives after the patient deceased was provided more frequently by GPs $(\mathrm{n}=175 ; 84.5 \%)$ than pulmonologists $(\mathrm{n}=20 ; 20.4 \%)$.

\section{Collaboration between healthcare providers}

When caring for palliative patients with COPD in the past year, pulmonologists indicated they most often collaborate with a specialized COPD-nurse in the hospital $(n=82$ answered often or always; $77.4 \%$ ) and GPs most often with a district nurse ( $\mathrm{n}=168$ answered often or always; 78.9\%) (Figure 3). According to the respondents, patients were most frequently referred to a physical therapist or dietician (Figure 4). Involvement of specialized palliative care providers varied. Almost one-third of pulmonologists $(n=33 ; 31.4 \%)$ reported that they often or always refer their palliative patients with COPD to a palliative care consultant; a quarter $(n=26 ; 24.8 \%)$ of them referred hardly any. A quarter of GPs ( $n=55 ; 25.6 \%)$ mentioned to collaborate often or always with a palliative care nurse and more than half never or rarely $(\mathrm{n}=117 ; 54.4 \%)$.
More than half of the pulmonologists $(\mathrm{n}=59 ; 52.7 \%)$ and GPs $(n=141 ; 61.8 \%)$ were satisfied with the collaboration between pulmonologists and GPs. Satisfaction about the information exchange between the hospital and primary care differed; more GPs were satisfied $(n=130$; $57.0 \%)$ than pulmonologists $(\mathrm{n}=34 ; 29.8 \%)$.

\section{Barriers to palliative care discussions}

The most frequently reported barrier to discuss palliative and end-of-life care topics with patients with COPD was the difficulty in predicting the disease course (Figure 5). The second reported barrier by pulmonologists was lack of time $(n=72 ; 63.7 \%)$, whereas only $14.8 \%(n=43)$ of the GPs mentioned this barrier. Lack of a clear definition of the palliative phase in COPD was more often indicated as a barrier by GPs than by pulmonologists ( $\mathrm{n}=158 ; 54.3 \%$ vs $\mathrm{n}=44 ; 38.9 \%, p=0.008)$. Further, patients' difficulties to specify what future care they want in case of disease deterioration was named as a barrier by both groups $(\mathrm{n}=88$; $30.2 \%$ of GPs and $n=47 ; 41.6 \%$ of pulmonologists).

\section{Discussion}

This is the first study giving a comprehensive overview of the palliative care provision for patients with COPD in primary and secondary care in the Netherlands. In contrast with previous studies conducted in other countries, ${ }^{3,14,15,16}$ we found that the majority of physicians regularly discuss 
palliative care topics with their patients with COPD. In a 2009 study, Dutch pulmonologists reported discussing life-sustaining treatments with $20 \%$ of their patients and life expectancy with $16 \%$; the rates found in our study are higher. ${ }^{17}$ Interestingly, we found that pulmonologists who had received a training in palliative care were more likely to discuss some important topics, such as spiritual needs, caregiver burden and end-of-life related topics than those without training. These findings may underscore the usefulness of palliative care education. Additionally, almost all participants, following guideline recommendations, reported to frequently prescribe opioids to treat dyspnea. In comparison, in a survey among Dutch pulmonologists in 2012, half of the participants hardly ever prescribed opioids in COPD patients with refractory dyspnea. ${ }^{19}$

It is plausible that the publication of the Dutch guideline in 2011, ${ }^{9}$ and to a lesser extent the Quality Framework in $2017,{ }^{10}$ may have had a positive impact on these care practices. Similarly, an increase in palliative care support was seen in the UK following the introduction of the End of Life Care strategy. ${ }^{18}$ Furthermore, a Dutch survey study in 2015 revealed that almost half of the pulmonologists reported no involvement of a specialist palliative care team to treat their COPD patients. ${ }^{7}$ Our study showed that this percentage had dropped considerably: only a quarter of pulmonologists hardly ever referred patients to a specialist palliative care team. This may be explained by the requirement for Dutch hospitals to have installed a specialist palliative care team since $2017 .^{20}$ Although this requirement concerns oncological palliative care, these teams can also be consulted for non-oncological patients. Involvement of such teams in the care of patients with COPD was found to have a positive effect on advance care planning documentation. $^{21}$

Despite these positive changes, palliative care for patients with COPD remains largely unstructured. The minority of respondents reported scheduling appointments specifically to discuss advance care planning. Furthermore, half of the responding pulmonologists and most GPs reported that their department or practice did not have any form of protocol nor specific agreements. This is comparable to findings from studies performed in the UK, Spain and Sweden. ${ }^{13,14,22}$ Moreover, some responding pulmonologists mentioned protocols related to palliative sedation and the dying phase, suggesting that in those cases palliative care is focused on terminal care only. Nevertheless, half of the pulmonologists and one-tenth of GPs said to have the intention to establish a protocol or agreements in the future, acknowledging the need to formalize palliative care in COPD. Though these intentions

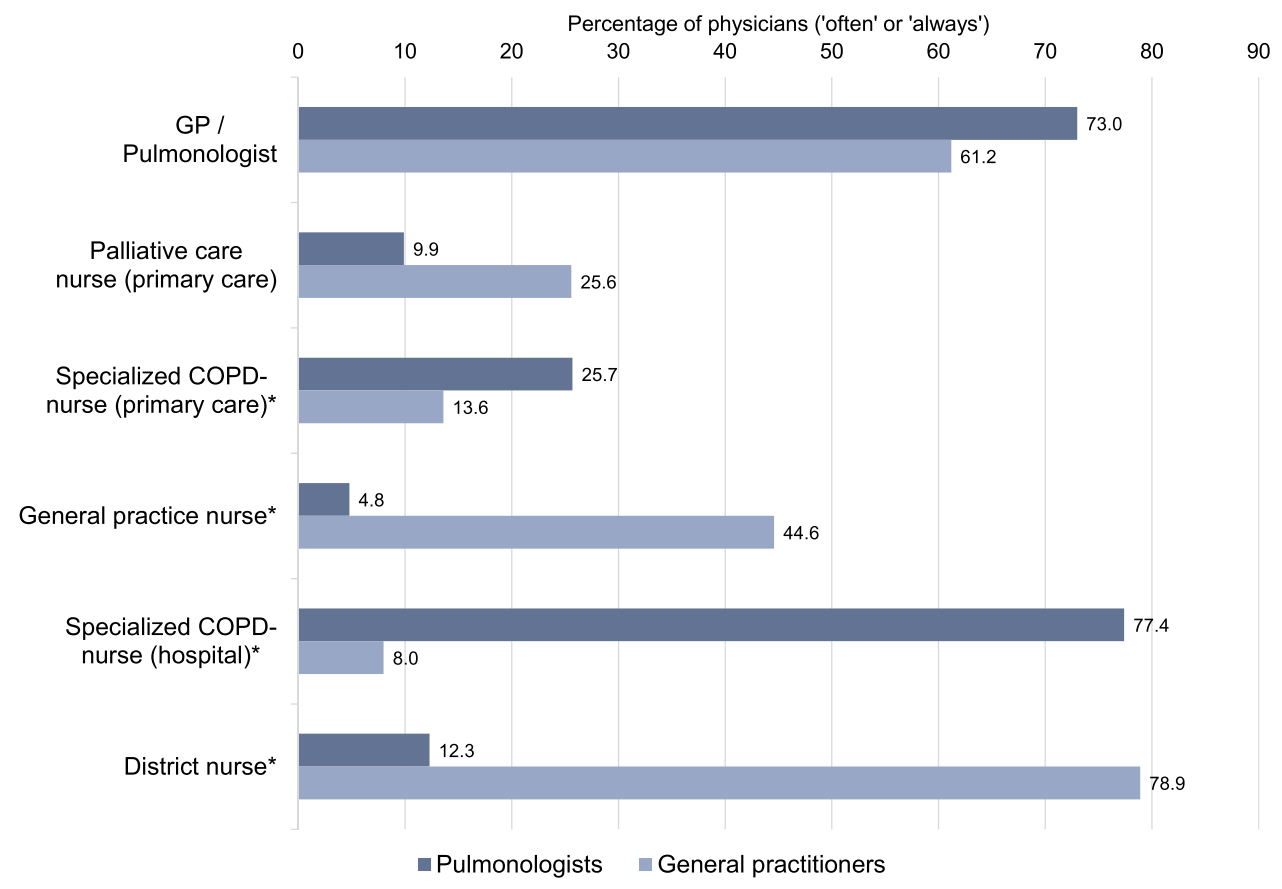

Figure 3 Collaboration with healthcare providers by pulmonologists and general practitioners. Percentages of physicians with answer often or always. *Significant difference ( $p<0.05$ using Mann-Whitney U-test).

Abbreviations: GP, general practitioner; PC, palliative care. 


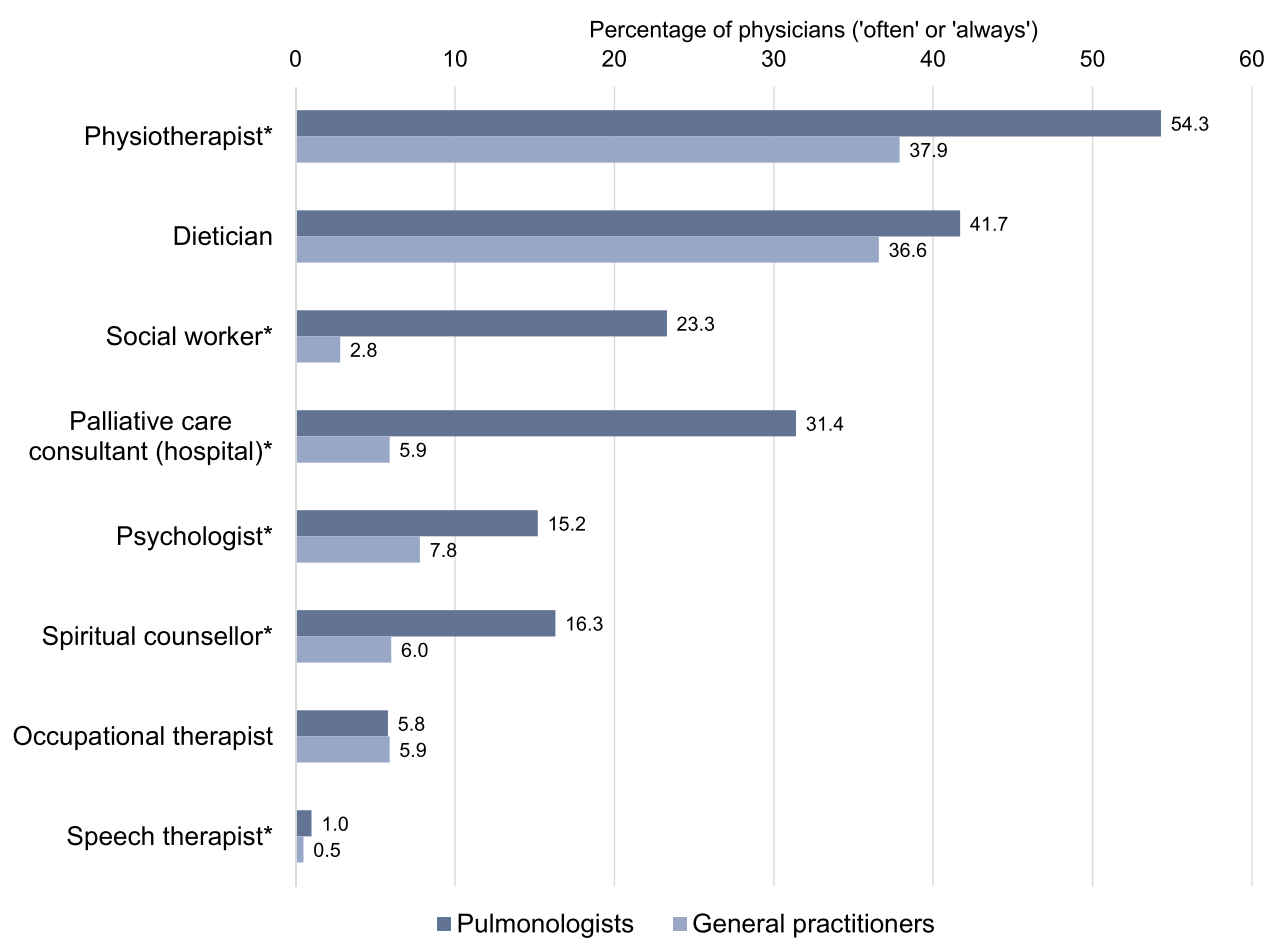

Figure 4 Referral to healthcare providers by pulmonologists and general practitioners. Percentages of physicians with answer often or always. *Significant difference $(p<$ 0.05 using Mann-Whitney $U$-test).

Abbreviation: PC, palliative care.

are promising, the explanations of their plans were nonspecific. As was highlighted earlier, guidelines provide insufficient clear guidance on when and how this care can be best provided. ${ }^{6}$ Therefore, more practical knowledge is needed. Examples may be a fixed time in the week scheduled for palliative care consultations, appointment of a care coordinator, clear criteria for (timely) involvement of specialist palliative care, and regular multidisciplinary meetings to discuss palliative patients with COPD.

GPs less frequently encountered a patient with COPD in the palliative phase than pulmonologists, but when they did, they discussed palliative care topics and scheduled these discussions more often than pulmonologists. These findings are not surprising, as advance care planning is considered by GPs a typical GP task. ${ }^{23}$ Further, the setting enables them to have these conversations at the patient's home. However, the risk is that patients with COPD with palliative care needs may not be timely recognized by GPs, since they have only one or two per year in their practice at most. GPs find advance care planning more challenging in patients with COPD and heart failure. ${ }^{23}$ Many GPs reported that they use the moment a pulmonologist informs the GP about the dire situation of the patient as a starting point. Together with the fact that in the Netherlands most patients with advanced COPD are under treatment of the pulmonologist, it may be more appropriate to consider the identification of patients with palliative care needs primarily the responsibility of a pulmonologist.

To identify those patients, the SQ was, next to clinical expertise, most often used by our participants. Noppe et al showed that using the SQ in recently hospitalized patients for an acute exacerbation of COPD is a useful and quick method. ${ }^{24}$ However, not all patients with palliative care needs were identified with this method. The structural use of symptom assessment tools such as the Edmonton Symptom Assessment Scale ${ }^{25}$ might have added value and could facilitate palliative care discussions with patients. Our results emphasize yet again that determining the most appropriate timing to start palliative care is difficult and perceived as an important barrier by most care providers. There is no consensus on when palliative care topics should be discussed and when specialist palliative care should be involved. ${ }^{6}$ We found that responding pulmonologists frequently held advance care planning discussions on the emergency department and during hospitalization. In an acute setting, these discussions may be limited to preferences regarding cardiopulmonary resuscitation and mechanical 


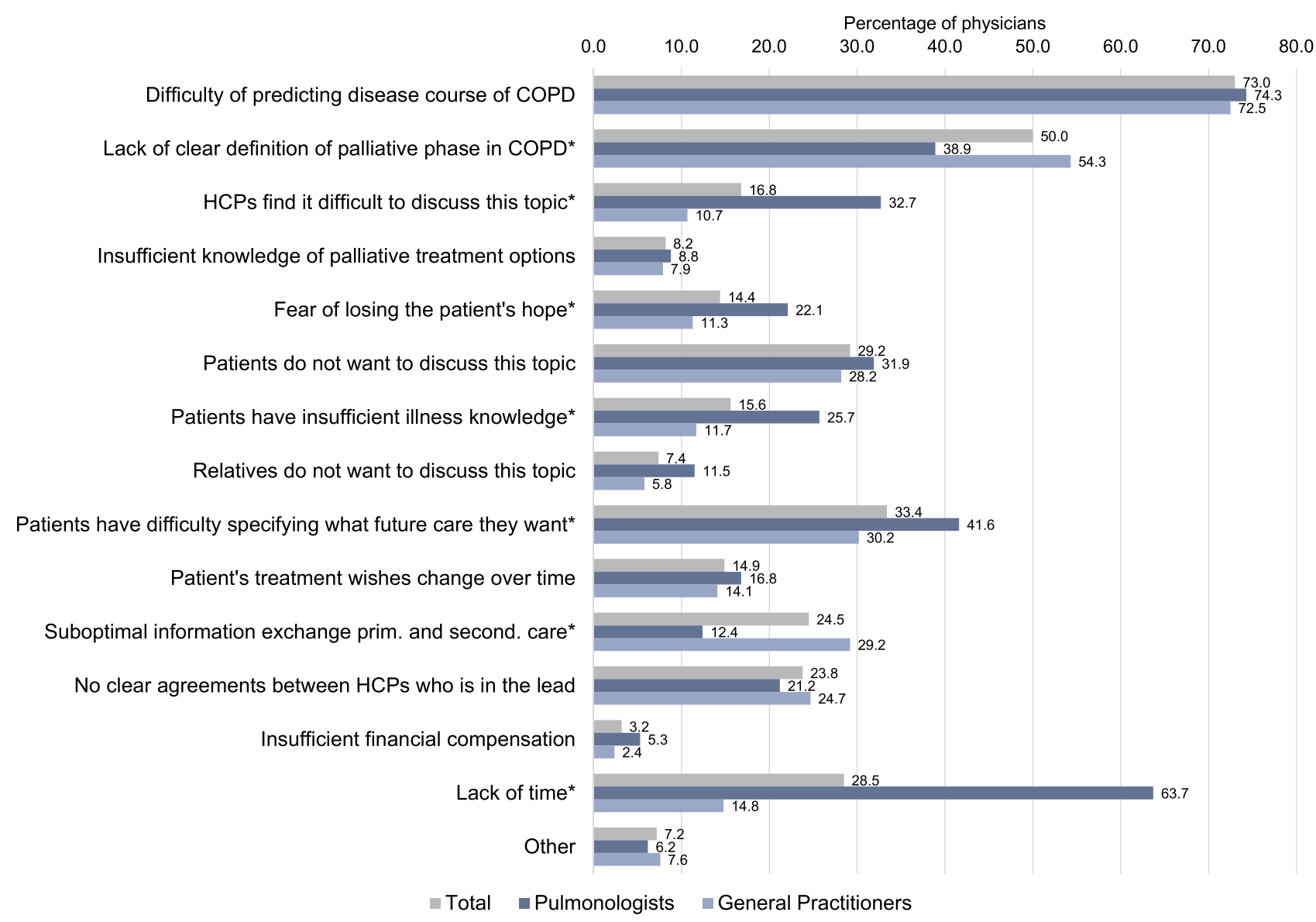

Figure 5 Barriers of palliative care discussions with patients with COPD, as indicated by pulmonologists, general practitioners and all respondents. *Significant difference between pulmonologists and GPs ( $p<0.05$ using Chi-square test).

Abbreviation: HCP, healthcare provider.

ventilation, while this is only one aspect of advance care planning. ${ }^{26}$ Additionally, it means that patients are confronted with these questions by a physician unfamiliar to them. During a planned visit to their regular physician in a non-acute setting, there is more opportunity to discuss the patient's wishes, values and preferences for future care with the patient and his/her family.

Since primary, as well as secondary care, are involved in COPD-care, a well-established collaboration between these settings is crucial. Although satisfaction about the collaboration between pulmonologists and GPs was reasonably high, information exchange between primary care and the hospital was viewed as problematic. This finding is consistent with previous literature. ${ }^{27}$ It is not merely a COPD specific problem: also letters from medical specialists to the GP about advanced cancer patients seldom contain advance care planning items. ${ }^{28}$ Establishing work agreements between pulmonologists and GPs may help to determine what and when to communicate. A shared medical record, whether integrated into an electronic medical record system or via a standardized paper form, is a prerequisite. After a pulmonologist identifies a patient with COPD in the palliative phase, contact should be initiated with his/her GP to discuss the patient's situation, options for future care and alignment of responsibilities.

\section{Limitations}

This study had some limitations. We asked participants to provide estimates on the frequency and content provided to patients with COPD in the palliative phase in the previous year. While this was a feasible and straightforward method to obtain an approximation of the provided care, it might not be an accurate reflection of the actually provided care because of recall bias and socialdesirability bias. Neither does it provide insights into the quality nor the timing of the provided care, e.g. whether treatments and discussions took place in the terminal or 
dying phase rather than early in the palliative phase. Although we achieved $76 \%$ coverage of pulmonology departments in the Netherlands, the response rate obtained among pulmonologists was lower than in previous studies. ${ }^{7,14,19}$ Also, we faced significant item nonresponse for unknown reasons. Additionally, respondents may be more concerned with palliative care than the general population of physicians, which could have influenced the results. Therefore, caution is warranted when extrapolating the results to all pulmonologists and GPs in the Netherlands. Finally, we did not include specialized COPD-nurses or advanced nurse practitioners in the study, even though they play an essential role in the care of patients with COPD. Their views and practices should be investigated in future research.

\section{Conclusions}

Most pulmonologists and GPs regularly discuss palliative care topics, use opioids to treat dyspnea and involve specialist palliative care consultants, probably with increasing frequency compared to a decade ago. However, palliative care for patients with COPD remains unstructured and little formalized, and advance care planning discussions frequently take place in an acute care setting. Nevertheless, there seems to be a high willingness to improve this care. To continue the upward trend, clear guidance and standardization of practice may help to decide when and how to initiate discussions, when to involve specialist palliative care and how to optimize information exchange between care settings. Furthermore, training in palliative care communication can empower healthcare providers to discuss end-of-life related topics, caregiver burden and spiritual needs.

\section{Abbreviations}

COPD, chronic obstructive pulmonary disease; GP, general practitioner; SQ, surprise question.

\section{Data Sharing Statement}

All relevant data are within the manuscript. Any other data are available upon request from the corresponding author.

\section{Acknowledgments}

The authors would like to thank Mirjam Riedijk for her contribution in collecting the data.

\section{Author Contributions}

All authors made substantial contributions to conception and design, acquisition of data, or analysis and interpretation of data; took part in drafting the article or revising it critically for important intellectual content; agreed to submit to the current journal; gave final approval of the version to be published; and agree to be accountable for all aspects of the work.

\section{Funding}

The authors disclosed receipt of the following financial support for the research, authorship and/or publication of this article: This work was financed by The Netherlands Organization for Health Research and Development (ZonMw) (project number: 844001401).

\section{Disclosure}

The authors declared no potential conflicts of interest with respect to the research, authorship and/or publication of this article.

\section{References}

1. Lozano R, Naghavi M, Foreman K, et al. Global and regional mortality from 235 causes of death for 20 age groups in 1990 and 2010: a systematic analysis for the Global Burden of Disease Study 2010. Lancet. 2012;380(9859):2095-2128. doi:10.1016/s0140-6736(12) 61728-0

2. Janssen DJ, Spruit MA, Wouters EF, Schols JM. Daily symptom burden in end-stage chronic organ failure: a systematic review. Palliat Med. 2008;22(8):938-948. doi:0269216308096906

3. Tavares N, Jarrett N, Hunt K, Wilkinson T. Palliative and end-of-life care conversations in COPD: a systematic literature review. ERJ Open Res. 2017;3(2):00068-2016. doi:10.1183/23120541.00068-2016

4. Beernaert K, Cohen J, Deliens L, et al. Referral to palliative care in COPD and other chronic diseases: a population-based study. Respir Med. 2013;107(11):1731-1739. doi:10.1016/j.rmed.2013.06.003

5. Momen N, Hadfield P, Kuhn I, Smith E, Barclay S. Discussing an uncertain future: end-of-life care conversations in chronic obstructive pulmonary disease. A systematic literature review and narrative synthesis. Thorax. 2012;67 (9):777-780. doi:10.1136/thoraxjnl-2012-201835

6. Siouta N, van Beek K, Preston N, et al. Towards integration of palliative care in patients with chronic heart failure and chronic obstructive pulmonary disease: a systematic literature review of European guidelines and pathways. BMC Palliat Care. 2016;15:18. doi:10.1186/s12904-016-0089-4

7. Duenk RG, Verhagen C, Dekhuijzen P, Vissers K, Engels Y, Heijdra Y. The view of pulmonologists on palliative care for patients with COPD: a survey study. Int J Chron Obstruct Pulmon Dis. 2017;12:299-311. doi:10.2147/copd.s121294

8. Siouta N, Clement P, Aertgeerts B, Van Beek K, Menten J. Professionals' perceptions and current practices of integrated palliative care in chronic heart failure and chronic obstructive pulmonary disease: a qualitative study in Belgium. BMC Palliat Care. 2018;17 (1):103. doi:10.1186/s12904-018-0356-7

9. Long Alliantie Nederland. Richtlijn Palliatieve zorg voor mensen met COPD. Long Alliantie Nederland. 2011. 
10. Boddaert M. Netherlands Quality Framework for Palliative Care. IKNL/Palliactief; 2017.

11. World Health Organization. WHO Definition of Palliative Care; 2002. Available from: http://www.who.int/cancer/palliative/defini tion/en/. Accessed December 12, 2018.

12. Veigh CM, Reid J, Larkin P, Porter S, Hudson P. The provision of generalist and specialist palliative care for patients with non-malignant respiratory disease in the North and Republic of Ireland: a qualitative study. BMC Palliat Care. 2017;17(1):6. doi:10.1186/s12904-017-0220-1

13. Roberts CM, Seiger A, Buckingham RJ, Stone RA. Clinician perceived good practice in end-of-life care for patients with COPD. Palliat Med. 2008;22(7):855-858. doi:10.1177/0269216308096722

14. Ecenarro PS, Iguiniz MI, Tejada SP, et al. Management of COPD in end-of-life care by Spanish pulmonologists. COPD. 2018;15 (2):171-176. doi:10.1080/15412555.2018.1441274

15. Gaspar C, Alfarroba S, Telo L, Gomes C, Barbara C. End-of-life care in COPD: a survey carried out with Portuguese pulmonologists. Rev Port Pneumol. 2014;20(3):123-130. doi:10.1016/j.rppneu.2014.01.008

16. Brożek B, Damps-Konstańska I, Pierzchała W, et al. End-of-life care for patients with advanced lung cancer and chronic obstructive pulmonary disease: survey among Polish pulmonologists. Pol Arch Intern Med. 2019;129(4):242-252. doi:10.20452/pamw.4478

17. Janssen DJA, Spruit MA, Schols J, Wouters EFM. A call for high-quality advance care planning in outpatients with severe COPD or chronic heart failure. Chest. 2011;139(5):1081-1088. doi:10.1378/chest.10-1753

18. Bloom CI, Slaich B, Morales DR, Smeeth L, Stone P, Quint JK. Low uptake of palliative care for COPD patients within primary care in the UK. Eur Respir J. 2018;51(2):1701879. doi:10.1183/13993003.01879-2017

19. Janssen DJ, de Hosson SM, Bij de Vaate E, Mooren KJ, Baas AA. Attitudes toward opioids for refractory dyspnea in COPD among Dutch chest physicians. Chron Respir Dis. 2015;12(2):85-92. doi: $10.1177 / 1479972315571926$

20. SONCOS. Standardisation of Multidisciplinary Cancer Care in the Netherlands. SONCOS Standardisation Report 5. 2017.
21. Duenk RG, Verhagen C, Bronkhorst EM, et al. Proactive palliative care for patients with COPD (PROLONG): a pragmatic cluster controlled trial. Int J Chron Obstruct Pulmon Dis. 2017;12:2795-2806. doi:10.2147/COPD.S141974

22. Strang S, Ekberg-Jansson A, Strang P, Larsson LO. Palliative care in COPD-web survey in Sweden highlights the current situation for a vulnerable group of patients. Ups J Med Sci. 2013;118 (3):181-186. doi:10.3109/03009734.2013.801059

23. Wichmann AB, van Dam H, Thoonsen B, Boer TA, Engels Y, Groenewoud AS. Advance care planning conversations with palliative patients: looking through the GP's eyes. BMC Fam Pract. 2018;19(1):184. doi:10.1186/s12875-018-0868-5

24. Noppe D, Veen HI, Mooren K. COPD patients in need of palliative care: identification after hospitalization through the surprise question. Chron Respir Dis. 2019;16:1479972318796219. doi:10.1177/ 1479972318796219

25. Chang VT, Hwang SS, Feuerman M. Validation of the edmonton symptom assessment scale. Cancer. 2000;88(9):2164-2171. doi:10.1002/ (sici)1097-0142(20000501)88:9<2164::aid-cncr24>3.0.co;2-5

26. Rietjens JAC, Sudore RL, Connolly M, et al. definition and recommendations for advance care planning: an international consensus supported by the European Association for palliative care. Lancet Oncol. 2017;18(9):e543-e551. doi:10.1016/s1470-2045(17)30582-x

27. Herrmann A, Carey ML, Zucca AC, Boyd LAP, Roberts BJ. Australian GPs' perceptions of barriers and enablers to best practice palliative care: a qualitative study. BMC Palliat Care. 2019;18(1):90. doi:10.1186/s12904-019-0478-6

28. Ermers DJM, van Bussel KJH, Perry M, Engels Y, Schers HJ. Advance care planning for patients with cancer in the palliative phase in Dutch general practices. Fam Pract. 2019;36(5):587-593. doi:10.1093/fampra/cmy124

\section{Publish your work in this journal}

The International Journal of COPD is an international, peer-reviewed journal of therapeutics and pharmacology focusing on concise rapid reporting of clinical studies and reviews in COPD. Special focus is given to the pathophysiological processes underlying the disease, intervention programs, patient focused education, and self management protocols. This journal is indexed on PubMed Central, MedLine and CAS. The manuscript management system is completely online and includes a very quick and fair peer-review system, which is all easy to use. Visit http://www.dovepress.com/testimonials.php to read real quotes from published authors.

Submit your manuscript here: https://www.dovepress.com/international-journal-of-chronic-obstructive-pulmonary-disease-journal 